\title{
A New Class of Function with Finitely Many Fixed Points
}

\author{
Matthew O. Oluwayemi $\mathbb{D}^{1,2}$ and Olubunmi A. Fadipe-Joseph ${ }^{3}$ \\ ${ }^{1}$ Landmark University SDG 4 (Quality Education Research Group), Omu-Aran, Nigeria \\ ${ }^{2}$ Department of Mathematics, Landmark University, P.M.B., 1001 Omu-Aran, Nigeria \\ ${ }^{3}$ Department of Mathematics, University of Ilorin, P.M.B., 1515 Ilorin, Nigeria
}

Correspondence should be addressed to Matthew O. Oluwayemi; oluwayemimatthew@gmail.com

Received 8 March 2021; Accepted 7 July 2021; Published 10 February 2022

Academic Editor: Alberto Fiorenza

Copyright (c) 2022 Matthew O. Oluwayemi and Olubunmi A. Fadipe-Joseph. This is an open access article distributed under the Creative Commons Attribution License, which permits unrestricted use, distribution, and reproduction in any medium, provided the original work is properly cited.

The study introduced a generalized multiplier operator used as a tool to define and investigate a new class of function, TS

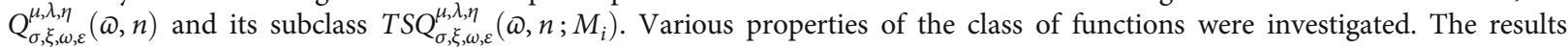
extend some known results in literature.

\section{Introduction and Preliminaries}

Let

$$
f(z)=z+\sum_{k=2}^{\infty} a_{k} z^{k}
$$

which are analytic in the open unit disk $\mathbb{U}=\{z \in \mathbb{C}:|z|<1\}$ and normalized by $f(0)=0$ and $f^{\prime}(0)=1$ be denoted by $S$.

We denote by $T$ the subclass of $S$ which are normalized univalent function of the form

$$
f(z)=z-\sum_{k=2}^{\infty} a_{k} z^{k}, \quad a_{k}>0,
$$

in the open unit disk $\mathbb{U}$. The function of the form (2) was first introduced in [1].

1.1. Differential Operators (Multiplier Transformations). In 1975, Ruscheweyh with the use of convolution introduced a differential operator $D^{n}$ as a tool to investigate a certain class of univalent functions. The continuous extension and generalization of the tool have since then continue to gain attention among the function theorists. The differential operator and some of such extensions are discussed as follows.
1.1.1. Ruscheweyh Differential Operator (1975). Let $n \in \mathbb{N}_{0}$ $=\{0,1,2, \cdots\}$. The Ruscheweyh derivative of $n^{\text {th }}$ order denoted by $R^{n} f(z)$ is defined by

$$
R^{n} f(z)=z+\sum_{k=2}^{\infty} B_{k}(n) a_{k} z^{k}
$$

where

$$
\begin{aligned}
B_{k} & =B(n, k)=\left(\begin{array}{c}
n+k-1 \\
n
\end{array}\right)=\frac{(n+1)(n+2) \cdots(n+k-1)}{(k-1) !} \\
& =\frac{(n+1)(n+2) \cdots(n+k-1)}{(k-1) !}=\frac{(n+1)_{k-1}}{(1)_{k-1}} .
\end{aligned}
$$

Hence,

$$
B(0, k)=\left(\begin{array}{c}
k-1 \\
0
\end{array}\right)=\frac{(n+1)_{k-1}}{(1)_{k-1}}=1
$$

So that $R^{0} f(z)$ is of the form (1).

1.1.2. Salagean Differential Operator (1983). Salagean defined an operator $D^{n}$ as follows. 
Let $f(z) \in A$, the differential operator $D^{n} f(z)$ is defined as

$$
D^{n} f(z)=z+\sum_{k=2}^{\infty} k^{n} a_{k} z^{k}, \quad n \in \mathbb{N}_{0} .
$$

Since the introduction of differential operators in geometric function theory by Ruscheweyh in [2] and Salagean in [3], several attempts have been made to extend and generalize the operators by various authors and researchers in the field. According to Aldawish et al. in [4], the differential operator forms the link between function theory and mathematical physics. Perhaps, this is the reason why the study of differential operators is growing interest in Geometric Functions Theory and Application (GFTA). Some of the numerous differential operators (multiplier transformations) established by some authors are found in [5-11].

The Salagean differential operator (6) defined in [3] appears to be the most famous and most referred to in general.

1.2. A Generalized Multiplier Transformation. In this work, a generalized operator is defined and used to investigate the class $\operatorname{TSQ}_{\sigma, \xi, \omega, \varepsilon}^{\mu, \lambda, \eta}(\omega, n)$ and its subclass $\operatorname{TSQ}_{\sigma, \xi, \omega, \varepsilon}^{\mu, \lambda, \eta}\left(\omega, n ; M_{i}\right)$ with their geometric properties. The results improve some existing results in literature as pointed out in the work. The work was motivated by [12].
Let $\mu, \lambda, \eta, \sigma, \xi, \varepsilon \geq 0$ with $\omega>0$ such that $\xi<\omega$ and $n \epsilon$ $\mathbb{N}_{0}=\mathbb{N} \cup\{0\}$. For $f \in S$, we define the following differential operator as follows:

$$
D_{\mu, \lambda, \eta}^{0}(\sigma, \xi, \omega, \varepsilon) f(z)=f(z)
$$

$$
\begin{aligned}
D_{\mu, \lambda, \eta}^{1}(\sigma, \xi, \omega, \varepsilon) f(z)= & \left(1-\frac{\lambda(\omega-\xi)^{\sigma}}{\mu+\omega}\right) f(z)+\frac{\lambda(\omega-\xi)^{\sigma}}{\mu+\omega} z f^{\prime}(z) \\
& +\frac{\eta+\varepsilon}{\mu+\omega} z^{2} f^{\prime \prime}(z), \\
\left(1-\frac{\lambda(\omega-\xi)^{\sigma}}{\mu+\omega}\right) f(z)= & \left(1-\frac{\lambda(\omega-\xi)^{\sigma}}{\mu+\eta}\right) z \\
& +\sum_{k=2}^{\infty}\left(1-\frac{\lambda(\omega-\xi)^{\sigma}}{\mu+\omega}\right) a_{k} z^{k}, \\
\left(\frac{\lambda(\omega-\xi)^{\sigma}}{\mu+\omega}\right) z f^{\prime}(z)= & \left(\frac{\lambda(\omega-\xi)^{\sigma}}{\mu+\omega}\right) z+\sum_{k=2}^{\infty} k\left(\frac{\lambda(\omega-\xi)^{\sigma}}{\mu+\omega}\right) a_{k} z^{k},
\end{aligned}
$$

$$
\left(\frac{\eta+\varepsilon}{\mu+\omega}\right) z^{2} f^{\prime \prime}(z)=\sum_{k=2}^{\infty}\left(\frac{k(k-1)(\delta+\varepsilon)}{\mu+\omega}\right) a_{k} z^{k} .
$$

Substituting (9), (10), and (11) in (8), we have

$$
\begin{aligned}
& D_{\mu, \lambda, \eta}^{1}(\sigma, \xi, \omega, \varepsilon) f(z)=z+\sum_{k=2}^{\infty}\left\{1-\frac{\lambda(\omega-\xi)^{\sigma}}{\mu+\omega}+\frac{k \lambda(\omega-\xi)^{\sigma}}{\mu+\omega}+\frac{k(k-1)(\eta+\varepsilon)}{\mu+\omega}\right\} a_{k} z^{k} \\
& D_{\mu, \lambda, \eta}^{1}(\sigma, \xi, \omega, \varepsilon) f(z)=z+\sum_{k=2}^{\infty}\left(1+\frac{(k-1)\left[\lambda(\omega-\xi)^{\sigma}+k(\eta+\varepsilon)\right]}{\mu+\omega}\right) a_{k} z^{k}
\end{aligned}
$$

Similarly,

$$
D_{\mu, \lambda, \eta}^{n}(\sigma, \xi, \omega, \varepsilon) f(z)=z\left(D_{\mu, \lambda, \eta}^{n-1}(\sigma, \xi, \omega, \varepsilon) f(z)\right) .
$$

Thus,

$$
\begin{aligned}
& D_{\mu, \lambda, \eta}^{n}(\sigma, \xi, \omega, \varepsilon) f(z)=z \\
& \quad+\sum_{k=2}^{\infty}\left(1+\frac{(k-1)\left[\lambda(\omega-\xi)^{\sigma}+k(\eta+\varepsilon)\right]}{\mu+\omega}\right)^{n} a_{k} z^{k}
\end{aligned}
$$

For convenience, for $f \in A$, we let

$$
D_{\mu, \lambda, \eta}^{n}(\sigma, \xi, \omega, \varepsilon) f(z)=z+\sum_{k=2}^{\infty} \Phi(n, k) a_{k} z^{k}
$$

where $\Phi(n, k)=\left(1+(k-1)\left[\lambda(\omega-\xi)^{\sigma}+k(\eta+\varepsilon)\right] / \mu+\omega\right)^{n}$.
Similarly, if $f \in T$ as defined in (2), then

$$
\begin{aligned}
& D_{\mu, \lambda, \eta}^{n}(\sigma, \xi, \omega, \varepsilon) f(z) \\
& \quad=z-\sum_{k=2}^{\infty}\left(1+\frac{(k-1)\left[\lambda(\omega-\xi)^{\sigma}+k(\eta+\varepsilon)\right]}{\mu+\omega}\right)^{n} a_{k} z^{k} \\
& D_{\mu, \lambda, \eta}^{n}(\sigma, \xi, \omega, \varepsilon) f(z)=z-\sum_{k=2}^{\infty} \Phi(n, k) a_{k} z^{k}
\end{aligned}
$$

where $\Phi(n, k)=\left(1+(k-1)\left[\lambda(\omega-\xi)^{\sigma}+k(\eta+\varepsilon)\right] / \mu+\omega\right)^{n}$.

The operator defined in (14) reduces to (1) when $n=0$. Furthermore, it reduces to some existing operators in literature. Setting some parameters as follows:

(i) For $\lambda=\omega=1$ and $\sigma=\mu=\varepsilon=\eta=0$, we obtain the Salagean operator in [3] 
(ii) When $\lambda=1, \mu=1, \sigma=0, \omega=1, \varepsilon=0$, and $\eta=0$, we get the Uralegaddi and Somanatha differential operator in [13]

(iii) The differential operator reduces to the Cho and Kim operator in [10] with $\lambda=1, \mu=1, \sigma=0, \varepsilon=$ 0 , and $\eta=0$

(iv) When $\mu=0, \omega=1, \sigma=0, \varepsilon=0$, and $\eta=0$, we get the Al-Oboudi differential operator in [14]

(v) When $\mu=1, \sigma=0, \varepsilon=0$, and $\eta=0$, we get multiplier transformation in [9]

(vi) Suppose $\mu=0, \omega=1, \sigma=0, \varepsilon=0, \eta=0$, and $\lambda=\alpha$ $+\delta / \gamma+\rho$ for $\alpha, \delta, \gamma, \rho \geq 0$ and provided that $\gamma+\rho$ $\neq 0$, then (14) reduces to the Alamri and Darus operator in [5]

(vii) When $\sigma=1$ and $\varepsilon=0$, we get the Amourah and Darus differential operator in [7]

(viii) Let $\lambda=\beta-\xi, \sigma=1, \varepsilon=0$, and $\eta=0$, we have the Amourah and Yousef differential operator in [8] provided $\beta>\xi$

In [15-17], the authors defined and studied different classes of univalent functions. Motivated by the work of Varma and Rosy in [12], we define the following class of univalent functions.

Definition 1. Let $\omega \geq 0$, a function $f$ defined by (2) belongs to the class $S Q_{\sigma, \xi, \omega, \varepsilon}^{\mu, \lambda, \eta}(\omega, n)$ if it satisfies the following condition:

$$
\begin{aligned}
& \operatorname{Re}\left\{\frac{D_{\mu, \lambda, \eta}^{n}(\sigma, \xi, \omega, \varepsilon) f(z)}{z}\right\} \\
& \quad \geq \omega\left|\left[D_{\mu, \lambda, \eta}^{n}(\sigma, \xi, \omega, \varepsilon) f(z)\right]^{\prime}-\frac{D_{\mu, \lambda, \eta}^{n}(\sigma, \xi, \omega, \varepsilon) f(z)}{z}\right|, \quad \omega \geq 0 .
\end{aligned}
$$

Investigation of univalent function is based on various classes and their geometric properties some of which are given in the following theorems and definitions.

Let $T \cap S Q_{\sigma, \xi, \omega, \varepsilon}^{\mu, \lambda, \eta}(\omega, n)=T S Q_{\sigma, \xi, \omega, \varepsilon}^{\mu, \lambda, \eta}(\omega, n)$. The necessary and sufficient condition for the functions in $S$ to be in TS $Q_{\sigma, \xi, \omega, \varepsilon}^{\mu, \lambda, \eta}(\omega, n)$ is investigated.

Remark 2. Let $T S Q_{\sigma, \xi, \omega, \mathcal{\varepsilon}}^{\mu, \lambda, \eta}\left(\omega, n ; M_{i}\right) \subset S Q_{\sigma, \xi, \omega, \mathcal{\varepsilon}}^{\mu, \lambda, \eta}(\omega, n)$. Then,

(i) $S Q_{\sigma, \xi, \omega, \mathcal{\varepsilon}}^{\mu, \lambda, \eta}(\omega, 0) \equiv S D(\alpha)$ and $T S Q_{\sigma, \xi, \omega, \mathcal{\varepsilon}}^{\mu, \lambda, \eta}\left(\omega, 0 ; M_{i}\right) \equiv T$ $S D(\alpha)$ studied by both Varma and Rosy and [12, 18]. The class of functions investigated in the work generalized some known classes of functions in literature

(ii) Similar classes of functions were also investigated in [19-21]

(iii) Different functions with finitely many fixed points were also considered in [22-24]

\section{Main Results}

Theorem 3. A function $f(z)$ defined by (16) is said to be in the class $\operatorname{TSQ}_{\sigma, \xi, \omega, \varepsilon}^{\mu, \lambda, \eta}(\omega, n)$ if and only if

$$
\sum_{k=2}^{\infty}\left(1+\frac{(k-1)\left[\lambda(\omega-\xi)^{\sigma}+k(\eta+\varepsilon)\right]}{\mu+\omega}\right)^{n}[1+\omega(k-1)]\left|a_{k}\right| \leq 1
$$

Proof. Suppose $f(z)$ defined by (16) satisfies (19). Then,

$$
\begin{aligned}
\operatorname{Re}\{ & \left.\frac{D_{\mu, \lambda, \eta}^{n}(\sigma, \xi, \omega, \varepsilon) f(z)}{z}\right\} \\
- & \omega\left|\left[D_{\mu, \lambda, \eta}^{n}(\sigma, \xi, \omega, \varepsilon) f(z)\right]^{\prime}-\frac{D_{\mu, \lambda, \eta}^{n}(\sigma, \xi, \omega, \varepsilon) f(z)}{z}\right| \\
& =1-\left|\left(1-\sum_{k=2}^{\infty} \Phi(n, k) a_{k} z^{k-1}\right)-\right| \\
- & \omega\left|\left(1-\sum_{k=2}^{\infty} k \Phi(n, k) a_{k} z^{k-1}\right)-\left(1-\sum_{k=2}^{\infty} \Phi(n, k) a_{k} z^{k-1}\right)\right| \\
& =1-\left|\left(\sum_{k=2}^{\infty} \Phi(n, k) a_{k} z^{k-1}\right)\right| \\
- & \oplus\left|1-\sum_{k=2}^{\infty} k \Phi(n, k) a_{k} z^{k-1}-1+\sum_{k=2}^{\infty} \Phi(n, k) a_{k} z^{k-1}\right| \\
& =1-\left|\sum_{k=2}^{\infty} \Phi(n, k) a_{k} z^{k-1}\right| \\
- & \oplus\left|\sum_{k=2}^{\infty}(k-1) \Phi(n, k) a_{k} z^{k-1}\right|, \quad|z|=r<1 \Rightarrow \leq 1 \\
- & \sum_{k=2}^{\infty}|\Phi(n, k)|\left|a_{k}\right|-\omega \sum_{k=2}^{\infty}|\Phi(n, k)|[1+\omega(k-1)]\left|a_{k}\right| \geq 0 .
\end{aligned}
$$

Hence, $f \in T S Q_{\sigma, \xi, \omega, \varepsilon}^{\mu, \lambda, \eta}\left(\omega, M_{j}\right)$.

Conversely,

$$
\begin{aligned}
& \operatorname{Re}\left\{\frac{D_{\mu, \lambda, \eta}^{n}(\sigma, \xi, \omega, \varepsilon) f(z)}{z}\right\} \\
& \quad-\omega\left|\left[D_{\mu, \lambda, \eta}^{n}(\sigma, \xi, \omega, \varepsilon) f(z)\right]^{\prime}-\frac{D_{\mu, \lambda, \eta}^{n}(\sigma, \xi, \omega, \varepsilon) f(z)}{z}\right|>0,
\end{aligned}
$$

which implies that $\operatorname{Re}\left\{1-\sum_{k=2}^{\infty}|\Phi(n, k)|\left|a_{k}\right|\right\}-\omega \sum_{k=2}^{\infty}(k-1$ )$|\Phi(n, k)|\left|a_{k}\right|>0$. 
Letting $z$ take real values and as $|z| \longrightarrow 1$, we get

$$
1-\sum_{k=2}^{\infty}|\Phi(n, k)|\left|a_{k}\right|-\omega \sum_{k=2}^{\infty}|\Phi(n, k)|(k-1)\left|a_{k}\right| \geq 0
$$

which implies $\sum_{k=2}^{\infty}|\Phi(n, k)|[1+\omega(k-1)]\left|a_{k}\right| \leq 1$ following (17).

Remark 4. For any function $f \in S Q_{\sigma, \xi, \omega, \mathcal{\varepsilon}}^{\mu, \lambda, \eta}(\omega, n)$,

$a_{k} \leq \frac{1}{\left(1+(k-1)\left[\lambda(\omega-\xi)^{\sigma}+k(\eta+\varepsilon)\right] / \mu+\omega\right)^{n}[1+\omega(k-1)]}, \quad k \geq 2$

The equality holds for

$f(z)=z-\sum_{k=2}^{\infty} \frac{1}{\left(1+(k-1)\left[\lambda(\omega-\xi)^{\sigma}+k(\eta+\varepsilon)\right] / \mu+\omega\right)^{n}[1+\omega(k-1)]} z^{k}$.

The class is the generalization of the class $\operatorname{TS} D(\alpha)$ investigated in $[12,18,25]$.

Corollary 5. A function $f(z)$ defined by (16) belongs to the class $f \in S Q_{\sigma, \xi, \omega, \varepsilon}^{\mu, \lambda, \eta}(\omega, 0)$ if

$$
\sum_{k=2}^{\infty}[1+\omega(k-1)]\left|a_{k}\right| \leq 1, \omega \geq 0
$$

The class $S Q_{\sigma, \xi, \omega, \varepsilon}^{\mu, \lambda, \eta}(\omega, 0) \equiv S D(\alpha)$ was investigated in [12, 18, 25].

Remark 6. Let $n=0$. Then, $f \in S Q_{\sigma, \xi, \omega, \mathcal{\varepsilon}}^{\mu, \lambda, \eta}(\omega, 0)$ with

$$
a_{k} \leq \frac{1}{1+\omega(k-1)}, \quad k \geq 2
$$

The equality holds for

$$
f(z)=z-\sum_{k=2}^{\infty} \frac{1}{1+\omega(k-1)} z^{k}
$$

See $[12,18,25]$ and the articles cited therein for details.

2.1. Subclass of Analytic Functions with Finitely Many Fixed Coefficients. We now introduce the class of $\operatorname{TSQ}_{\sigma, \xi, \omega, \varepsilon}^{\mu, \lambda, \eta}(\omega, n$; $\left.M_{i}\right)$ as a subclass of $T S Q_{\sigma, \xi, \omega, \varepsilon}^{\mu, \lambda, \eta}(\Phi, n)$ with

$$
\begin{aligned}
f(z)= & z-\sum_{i=2}^{t} \frac{M_{i}}{\left(1+(i-1)\left[\lambda(\omega-\xi)^{\sigma}+i(\eta+\varepsilon)\right] / \mu+\omega\right)^{n}[1+\omega(i-1)]} z^{i} \\
& -\sum_{k=t+1}^{\infty} a_{k} z^{k} .
\end{aligned}
$$

In recent times, the authors in $[12,19-24,26]$ have also investigated classes of univalent functions with finitely many fixed coefficients.

Theorem 7. A function $f(z)$ defined by (28) is said to be in the class $T S Q_{\sigma, \xi, \omega, \varepsilon}^{\mu, \lambda, \eta}\left(\omega, n ; M_{i}\right)$ if

$$
\begin{aligned}
& \sum_{k=t+1}^{\infty}\left(1+\frac{(k-1)\left[\lambda(\omega-\xi)^{\sigma}+k(\eta+\varepsilon)\right]}{\mu+\omega}\right)^{n}[1+\omega(k-1)] a_{k} \\
& \quad \leq 1-\sum_{i=2}^{t} M_{i}
\end{aligned}
$$

where $\omega \geq 0,0 \leq M_{i} \leq 1$, and $0 \leq \sum_{i=2}^{t} M_{i} \leq 1$.

Proof. Suppose $f \in T S Q_{\sigma, \xi, \omega, \varepsilon}^{\mu, \lambda, \eta}\left(\omega, n ; M_{i}\right)$. Then, from (23),

$a_{i} \leq \frac{M_{i}}{\left(1+(i-1)\left[\lambda(\omega-\xi)^{\sigma}+j(\eta+\varepsilon)\right] / \mu+\omega\right)^{n}[1+\omega(i-1)]}$,

$$
i=2,3, \cdots, t, 0 \leq M_{i} \leq 1,0 \leq \sum_{i=2}^{t} M_{i} \leq 1,
$$

which implies $\quad \sum_{i=2}^{t} M_{i}+\sum_{k=j+1}^{\infty}$ $\left(1+(k-1)\left[\lambda(\omega-\xi)^{\sigma}+k(\eta+\varepsilon)\right] / \mu+\omega\right)^{n}[1+\omega(k-1)] a_{k} \leq 1$

Conversely,

$$
\begin{aligned}
\operatorname{Re} & \left\{\frac{D_{\mu, \lambda, \eta}^{n}(\sigma, \xi, \omega, \varepsilon) f(z)}{z}\right\} \\
& -\omega\left|\left[D_{\mu, \lambda, \eta}^{n}(\sigma, \xi, \omega, \varepsilon) f(z)\right]^{\prime}-\frac{D_{\mu, \lambda, \eta}^{n}(\sigma, \xi, \omega, \varepsilon) f(z)}{z}\right| \\
& \geq 1-\left|\frac{D_{\mu, \lambda, \eta}^{n}(\sigma, \xi, \omega, \varepsilon) f(z)}{z}-1\right| \\
& -\omega\left|\left[D_{\mu, \lambda, \eta}^{n}(\sigma, \xi, \omega, \varepsilon) f(z)\right]^{\prime}-\frac{D_{\mu, \lambda, \eta}^{n}(\sigma, \xi, \omega, \varepsilon) f(z)}{z}\right| \\
& =1-\sum_{k=2}^{\infty}\left(1+\frac{(k-1)\left[\lambda(\omega-\xi)^{\sigma}+k(\eta+\varepsilon)\right]}{\mu+\omega}\right)^{n}\left|a_{k}\right| \\
& -\omega \sum_{k=2}^{\infty}\left(1+\frac{(k-1)\left[\lambda(\omega-\xi)^{\sigma}+k(\eta+\varepsilon)\right]}{\mu+\omega}\right)^{n}(k-1)\left|a_{k}\right| \\
& =1-\sum_{i=2}^{t}\left(1+\frac{(i-1)\left[\lambda(\omega-\xi)^{\sigma}+k(\eta+\varepsilon)\right]}{\mu+\omega}\right)^{n}[1+\omega(i-1)]\left|a_{j}\right| \\
& -\sum_{k=i+1}^{\infty}\left(1+\frac{(k-1)\left[\lambda(\omega-\xi)^{\sigma}+k(\eta+\varepsilon)\right]}{\mu+\omega}\right)^{n}[1+\omega(k-1)]\left|a_{k}\right|=1 \\
& -\sum_{i=2}^{t} M_{i}-\sum_{k=i+1}^{\infty}\left(1+\frac{(k-1)\left[\lambda(\omega-\xi)^{\sigma}+k(\eta+\varepsilon)\right]}{\mu+\omega}\right)^{n}[1+\omega(k-1)]\left|a_{k}\right| \geq 0,
\end{aligned}
$$

by (29) and (30). Thus, $f \in T S Q_{\sigma, \xi, \omega, \varepsilon}^{\mu, \lambda, \eta}\left(\omega, n ; M_{i}\right)$. 
The result is sharp for

$$
\begin{aligned}
f(z)= & z-\sum_{i=2}^{t} \frac{M_{i}}{\left(1+(i-1)\left[\lambda(\omega-\xi)^{\sigma}+j(\eta+\varepsilon)\right] / \mu+\omega\right)^{n}[1+\omega(i-1)]} z^{i} \\
& -\frac{\left(1-\sum_{i=2}^{t} M_{j}\right)}{\left(1+(k-1)\left[\lambda(\omega-\xi)^{\sigma}+k(\eta+\varepsilon)\right] / \mu+\omega\right)^{n}[1+\omega(k-1)]} z^{k} .
\end{aligned}
$$

Corollary 8. Let $T S Q_{\sigma, \xi, \omega, \varepsilon}^{\mu, 0, \eta}\left(\omega, n ; M_{i}\right)$. Then,

$a_{k} \leq \frac{\left(1-\sum_{i=2}^{t} M_{i}\right)}{\left(1+(k-1)\left[\lambda(\omega-\xi)^{\sigma}+k(\eta+\varepsilon)\right] / \mu+\omega\right)^{n}[1+\omega(k-1)]}, \quad k \geq t+1$.

The result is sharp for $f(z)$ given by (32).

Corollary 9. For $\operatorname{TSQ}_{\sigma, \xi, \omega, \mathcal{E}}^{\mu, \lambda, \eta}\left(\omega, 0 ; M_{i}\right)$,

$$
a_{k} \leq \frac{1-\sum_{i=2}^{t} M_{i}}{[1+\omega(k-1)]}, \quad k \geq t+1 .
$$

The result is sharp for $f(z)$ given by

$$
f(z)=z-\sum_{i=2}^{t} \frac{M_{i}}{1+\omega(i-1)]} z^{i}-\frac{\left(1-\sum_{i=2}^{t} M_{i}\right)}{1+\omega(k-1)} z^{k}, \quad k \geq 1 .
$$

The class $\operatorname{TSQ}_{\sigma, \xi, \omega, \varepsilon}^{\mu, 0, \eta}\left(\omega, 0 ; M_{i}\right) \equiv \operatorname{TSD}(\alpha)$ was investigated in [12].

Theorem 10. The class $\operatorname{TSQ}_{\sigma, \xi, \omega, \varepsilon}^{\mu, \lambda, \eta}\left(\omega, n ; M_{i}\right)$ is convex.

Proof. Suppose there exist functions $f, g \in T S Q_{\sigma, \xi, \omega, \varepsilon}^{\mu, \lambda, \eta}(\omega, n$; $M_{i}$ ) such that

$$
\begin{aligned}
f(z)= & z-\sum_{i=2}^{t} \frac{M_{i}}{\left(1+(k-1)\left[\lambda(\omega-\xi)^{\sigma}+k(\eta+\varepsilon)\right] / \mu+\omega\right)^{n}[1+\omega(k-1)]} z^{i} \\
& -\sum_{i=t+1}^{\infty} a_{k} z^{k}, \\
g(z)= & z-\sum_{i=2}^{t} \frac{M_{i}}{\left(1+(k-1)\left[\lambda(\omega-\xi)^{\sigma}+k(\eta+\varepsilon)\right] / \mu+\omega\right)^{n}[1+\omega(k-1)]} z^{i} \\
& -\sum_{i=t+1}^{\infty} b_{k} z^{k},
\end{aligned}
$$

where $0 \leq M_{i} \leq 1$ and $0 \leq \sum_{i=2}^{t} M_{i} \leq 1$. Then, let $0 \leq \zeta \leq 1$ and

$$
h(z)=\zeta f(z)+(1-\zeta) g(z)
$$

So that by substituting (36) and (37) in (38), we have

$$
\begin{aligned}
h(z)= & z-\sum_{i=2}^{\infty} \frac{M_{i}}{\left(1+(k-1)\left[\lambda(\omega-\xi)^{\sigma}+k(\eta+\varepsilon)\right] / \mu+\omega\right)^{n}[1+\omega(k-1)]} z^{i} \\
& -\sum_{i=t+1}^{\infty}\left[\zeta a_{k}+(1-\zeta) b_{k}\right] z^{k} .
\end{aligned}
$$

Such that if $\zeta=1$ and $\zeta=0$, equation (39) reduces to (36) and (37), respectively. Now,

$$
\begin{aligned}
\sum_{k=i+1}^{\infty} & \left(1+\frac{(k-1)\left[\lambda(\omega-\xi)^{\sigma}+k(\eta+\varepsilon)\right]}{\mu+\omega}\right)^{n}[1+\omega(k-1)]\left[\zeta a_{k}+(1-\zeta) b_{k}\right] \\
= & \zeta \sum_{k=i+1}^{\infty}\left(1+\frac{(k-1)\left[\lambda(\omega-\xi)^{\sigma}+k(\eta+\varepsilon)\right]}{\mu+\omega}\right)^{n}[1+\omega(k-1)] a_{k} \\
& +(1-\zeta) \sum_{k=i+1}^{\infty}\left(1+\frac{(k-1)\left[\lambda(\omega-\xi)^{\sigma}+k(\eta+\varepsilon)\right]}{\mu+\omega}\right)^{n}[1+\omega(k-1)] b_{k} \\
\leq & \zeta\left(1-\sum_{i=2}^{t} M_{i}\right)+(1-\zeta)\left(1-\sum_{i=2}^{t} M_{i}\right)=1-\sum_{i=2}^{t} M_{i} .
\end{aligned}
$$

Thus, $h(z) \in \operatorname{TSQ}_{\sigma, \xi, \omega, \varepsilon}^{\mu, \lambda, \eta}\left(\omega, n ; M_{i}\right)$.

Theorem 11. Let

$$
\begin{aligned}
f_{t}(z)= & z-\sum_{i=2}^{t} \frac{M_{i}}{\left(1+(k-1)\left[\lambda(\omega-\xi)^{\sigma}+k(\eta+\varepsilon)\right] / \mu+\omega\right)^{n}[1+\omega(k-1)]} z^{i}, \\
f_{k}(z)= & z-\sum_{i=2}^{t} \frac{M_{i}}{\left(1+(i-1)\left[\lambda(\omega-\xi)^{\sigma}+i(\eta+\varepsilon)\right] / \mu+\omega\right)^{n}[1+\omega(i-1)]} z^{i} \\
& -\sum_{k=t+1}^{\infty} \frac{\left(1-\sum_{k=2}^{t} M_{i}\right)}{\left(1+(k-1)\left[\lambda(\omega-\xi)^{\sigma}+k(\eta+\varepsilon)\right] / \mu+\omega\right)^{n}[1+\omega(k-1)]} z^{k},
\end{aligned}
$$

$$
k \geq i+1
$$

Then, $f \in \operatorname{TSQ}_{\sigma, \xi, \omega, \varepsilon}^{\mu, \lambda, \eta}\left(\omega, n ; M_{i}\right)$ if and only if $f$ can be expressed in the form

$$
f(z)=\sum_{k=t}^{\infty} \zeta_{k} f_{k}(z)
$$

where $\zeta_{k} \geq 0$ with $k \geq t$ and $\sum_{k=t}^{\infty} \zeta_{k} f_{k}(z)=1$.

Proof. If $f \in T$ can be expressed in form of (42). Then,

$$
\begin{aligned}
f(z)= & z-\sum_{i=2}^{t} \frac{M_{i}}{\left(1+(i-1)\left[\lambda(\omega-\xi)^{\sigma}+i(\eta+\varepsilon)\right] / \mu+\omega\right)^{n}[1+\omega(i-1)]} z^{i} \\
& -\sum_{k=t+1}^{\infty} \frac{\zeta_{k}\left[1-\sum_{k=2}^{t} M_{i}\right]}{\left(1+(k-1)\left[\lambda(\omega-\xi)^{\sigma}+k(\eta+\varepsilon)\right] / \mu+\omega\right)^{n}[1+\omega(k-1)]} z^{k} .
\end{aligned}
$$


Now,

$$
\begin{aligned}
& \sum_{k=t+1}^{\infty}\left(1+\frac{(k-1)\left[\lambda(\omega-\xi)^{\sigma}+k(\eta+\varepsilon)\right]}{\mu+\omega}\right)^{n}[1+\omega(k-1)] \\
& \cdot\left\{\frac{\zeta_{k}\left[1-\sum_{k=2}^{t} M_{i}\right]}{\left(1+(k-1)\left[\lambda(\omega-\xi)^{\sigma}+k(\eta+\varepsilon)\right] / \mu+\omega\right)^{n}[1+\omega(k-1)]}\right\} \\
& \quad=\sum_{k=t+1}^{\infty} \zeta_{k}\left[1-\sum_{k=2}^{t} M_{i}\right]=\left[1-\sum_{k=2}^{t} M_{i}\right] \sum_{k=t+1}^{\infty} \zeta_{k} \\
& \quad=\left[1-\sum_{k=2}^{t} M_{i}\right]\left(1-\zeta_{k}\right) \leq 1-\sum_{k=2}^{t} M_{i},
\end{aligned}
$$

which implies $f \in \operatorname{TSQ}_{\sigma, \xi, \omega, \varepsilon}^{\mu, \lambda, \eta}\left(\varrho, n ; M_{i}\right)$.

Conversely, for $k \geq t+1$, set

$$
\zeta_{k}=\frac{\left(1+(k-1)\left[\lambda(\omega-\xi)^{\sigma}+k(\eta+\varepsilon)\right] / \mu+\omega\right)^{n}[1+\omega(k-1)] a_{k}}{1-\sum_{i=2}^{\infty} M_{i}}, \quad k \geq t+1,
$$

$$
\zeta_{t}=1-\sum_{k=t+1}^{\infty} \zeta_{k}
$$

Then, $f$ can be represented as $f(z)=\sum_{k=t}^{\infty} \zeta_{k} f_{k}(z)$.

\section{Conclusion}

In this work, a generalized multiplier operator was defined and used to define a class of univalent function. Geometric properties of the class $T S Q_{\sigma, \xi, \omega, \varepsilon}^{\mu, \lambda, \eta}(\varrho, n)$ and its subclass $T S$ $Q_{\sigma, \xi, \omega, \varepsilon}^{\mu, \lambda, \eta}\left(\varrho, n ; M_{i}\right)$ were investigated. The results obtained are the generalization of many known results in literature. See $[12,18-21]$.

\section{Data Availability}

No data was used.

\section{Conflicts of Interest}

The authors declare that there are no competing interests.

\section{Authors' Contributions}

Both authors contributed to the work and approved the final manuscript.

\section{Acknowledgments}

The first author would like to acknowledge Landmark University, Nigeria, for the support received.

\section{References}

[1] H. Silverman, "Univalent functions with negative coefficients," Proceedings of American Mathematical Society, vol. 51, no. 1, pp. 109-116, 1975.

[2] S. Ruscheweyh, "New criteria for univalent functions," Proceedings of American Mathematical Society, vol. 49, no. 1, pp. 109-115, 1975.

[3] G. S. Salagean, Subclasses of Univalent Functions, Complex Analysis-Fifth Romanian-Finnish Seminar, Springer, Berlin Heidelberg, 1983.

[4] M. Aldawish, M. Darus, and W. Ibrahim, "Concavity of Ruscheweyh differential operator," Acta Universitatis Apulensis, vol. 37, pp. 73-82, 2014.

[5] M. Alamri and M. Darus, "On univalent function with negative coefficients by using differential operator," AIP Conference Proceedings, vol. 1614, p. 871, 2014.

[6] A. G. Alamoush and M. Darus, "On certain subclasses of analytic multivalent functions using generalized Salagean operator," International Journal of Differential Equations, vol. 2015, 7 pages, 2015.

[7] A. Amourah and M. Darus, "Some properties of a new class of univalent functions involving a new generalized differential operator with negative coefficients," Indian Journal of Science and Technology, vol. 9, no. 36, pp. 1-7, 2016.

[8] A. A. Amourah and F. Yousef, "Some properties of a class of analytic functions involving a new generalized differential operator," Boletim da Sociedade Paranaense de Matematica, vol. 38, no. 6, pp. 33-42, 2020.

[9] A. Catas, On Certain Class of p-Valent Functions Defined by New Multiplier Transformations, Proceedings Book of the International Symposium on Geometric Function Theory and Applications, 20-24, 2007, TC Isambul Kultur University, Turkey, 2007.

[10] N. E. Cho and T. M. Kim, "Multiplier transformations and strongly close-to-convex functions," Bulletin of the Korean Mathematical Society, vol. 40, no. 3, pp. 399-410, 2003.

[11] S. R. Swamy, "On univalent functions defined by a new generalized multiplier differential operator," Journal of Mathematical and Computational Science, vol. 2, no. 2012, pp. 1233 1240, 2012.

[12] S. S. Varma and T. Rosy, "Certain properties of a subclass of univalent functions with finitely many fixed coefficients," Khayyam Journal Mathematics, vol. 1, no. 3, pp. 25-32, 2017.

[13] B. A. Uralegaddi and C. Somanatha, "Certain classes of univalent functions," in Current Topics in Analytic Function Theory, H. M. Srivastava and S. Owa, Eds., pp. 371-374, World Scientific Publishing Company, Singapore, 1992.

[14] F. M. Al-Oboudi, "On univalent functions defined by a generalized Sălăgean operator," International Journal of Mathematics and Mathematical Sciences, vol. 2004, no. 27, p. 1436, 2004.

[15] S. Kanas, Ş. Altinkaya, and S. Yalçin, "Subclass of k-uniformly starlike functions defined by the symmetric q-derivative operator," Ukrainian Mathematical Journal, vol. 70, no. 11, pp. 1727-1740, 2019.

[16] R. Bharati, R. Parvatham, and A. Swaminathan, "On subclasses of uniformly convex functions and corresponding class of starlike functions," Tamkang Journal of Mathematics, vol. 28, no. 1, pp. 17-32, 1997. 
[17] M. O. Oluwayemi and I. Faisal, "A new family of analytic functions associated with multiplier transformation," Scientific Africa, vol. 12, pp. 1-9, 2021.

[18] R. Ezhilarasi, T. V. Sudharsan, M. H. Mohd, and K. G. Subramanian, "Connections between certain subclasses of analytic univalent functions based on operators," Journal of Complex Analysis, vol. 2017, 5 pages, 2017.

[19] S. Porwal, K. K. Dixit, A. L. Pathak, and R. Tripathi, “A unified study of certain subclasses of $\alpha$-uniformly convex functions of order $\beta$ with negative coefficients," Gulf Journal of Mathematics, vol. 2, no. 4, pp. 94-105, 2014.

[20] M. Shanthi and C. Selvaraj, "A subclass of multivalent functions with finitely many fixed coefficients," International Journal of Pure and Applied Mathematics, vol. 118, no. 10, pp. 479489, 2018.

[21] K. V. Vidyasagar, "Geometric properties of some class of univalent functions by fixing finitely many coefficients," International Journal of Innovative Science, Engineering and Technology, vol. 7, no. 1, pp. 49-56, 2019.

[22] K. K. Dixit and I. B. Misra, "A class of uniformly convex functions of order $\alpha$ with negative and fixed finitely many coefficients," Indian Journal of Pure and Applied Mathematics, vol. 32, no. 5, pp. 711-716, 2001.

[23] M. O. Oluwayemi and J. O. Okoro, "Certain results on a class of functions with negative coefficients," International Journal of Mathematics and Computer Science, vol. 16, no. 4, pp. 1295-1302, 2021.

[24] S. Owa and H. M. Srivastava, "A class of analytic functions with fixed finitely many coefficients," Journal of Faculty of Science and Technology Kinki University, vol. 31, pp. 1-10, 1987.

[25] R. Ezhilarasi, T. V. Sudharsan, and S. Sivasubramanian, "On certain subclass of univalent functions with finitely many fixed coefficients defined by Bessel function," Malaya Journal of Matematik, vol. 8, no. 3, pp. 1085-1091, 2020.

[26] M. O. Oluwayemi, O. A. Fadipe-Joseph, and N. Sh, "On a subclass of analytic functions with fixed finitely many coefficients based on Salagean operator and modified sigmoid function," Advances in Mathematics: Scientific Journal, vol. 10, no. 6, pp. 2807-2820, 2021. 